\title{
Multilinguales
}

15 | 2021

Recherches algériennes en sociolinguistique et en littérature : discours scientifique/discours militant, quelles frontières?

\section{Une analyse discursive de l'acte humoristique à travers les créations lexicales dans la presse francophone algérienne}

A discursive analysis of the humorous act through lexical creations in the francophone Algerian press

\section{Samira Allam Iddou}

\section{(2) OpenEdition} Journals

Édition électronique

URL : https://journals.openedition.org/multilinguales/6199

DOI : $10.4000 /$ multilinguales.6199

ISSN : 2335-1853

Éditeur

Université Abderrahmane Mira - Bejaia

Référence électronique

Samira Allam Iddou, «Une analyse discursive de l'acte humoristique à travers les créations lexicales dans la presse francophone algérienne », Multilinguales [En ligne], 15|2021, mis en ligne le 15 juin 2021, consulté le 14 février 2022. URL : http://journals.openedition.org/multilinguales/6199; DOI : https://doi.org/10.4000/multilinguales.6199

Ce document a été généré automatiquement le 14 février 2022.

\section{(i) 89

Multilinguales est mise à disposition selon les termes de la Licence Creative Commons Attribution Pas d'Utilisation Commerciale - Pas de Modification 4.0 International 


\title{
Une analyse discursive de l'acte
} humoristique à travers les créations lexicales dans la presse francophone algérienne

\author{
A discursive analysis of the humorous act through lexical creations in the \\ francophone Algerian press
}

Samira Allam Iddou

1 Cet article s'inscrit dans la réflexion sur l'acte humoristique dans le discours journalistique francophone à travers les créations lexicales. Cette étude a pour but de mettre l'accent sur les procédés de création lexicale (dérivation, composition, inversion, synapsie, troncation ou siglaison, métaphore, emprunt,...) pour véhiculer l'humour. Si l'innovation lexicale a fait l'objet de beaucoup d'études, nous ne recensons pas de recherches consacrées à la création lexicale à effet humoristique de la presse francophone algérienne. Ce constat nous amène à poser la question suivante :

-Quels sont les procédés de création lexicale pour véhiculer l'humour dans le discours journalistique d'expression française?

2 A cet effet, nous nous permettons de mettre à jour une analyse discursive de l'humour par le biais des créations lexicales manifestées dans le discours journalistique. Dans le cadre de cette étude, nous proposons d'abord de relever toutes les créations pouvant servir de base à des effets humoristiques afin de les analyser ensuite, nous mettons en lumière la mise en œuvre du processus néologique. Cette étude entreprend d'illustrer dans cette perspective la typologie des néologismes proposée par Sablayrolles (2000) par des exemples tirés des chroniques journalistiques ${ }^{1}$. Ces lexies reposent sur différents procédés linguistiques (suffixation : bouteflikisme ${ }^{2}$; amalgame :démocrachie ${ }^{3}$; synapsies :pauvres de Bill Gates ${ }^{4}$, transcatégorisation: castinguent ${ }^{5}(\mathrm{n}>\mathrm{v}$, etc.). Des métaphores comme le Lénine du Funk $k^{6}$ semblent aussi propres au contexte ${ }^{7}$ de la presse algérienne. 


\section{Quelques caractéristiques générales de la presse écrite}

\subsection{La typologie des genres journalistiques}

3 La presse écrite a pour principale fonction l'Information, c'est-à-dire, la transmission de la grande et petite actualité, mais aussi l'expression des jugements, des idées et des opinions. J. De Brouker (1995: p. 207) distingue deux grandes familles de genres rédactionnels : les genres de l'information et les genres du commentaire. Les genres de l'information comprennent la brève, le reportage, la dépêche, etc. Tandis que les genres du commentaire comprennent la tribune, le billet, l'éditorial, la chronique, etc. Selon M. Voirol (1995 : p. 18), l'article d'information a pour principal objectif « de relater des évènements, de livrer des faits, de montrer les personnages ", l'article de commentaire a pour objet « de développer des idées, de livrer une opinion, d'affirmer une position. A cette catégorie appartiennent le billet, la critique, l'éditorial, la chronique, etc.

4 Au sujet des typologies des genres journalistiques, J-M. Adam (1997 : p. 3-18) les résume selon deux positions énonciatives polaires. La première concerne l'engagement $d u$ journaliste, nommé aussi la « distance ». C'est le cas de l'article d'information (la brève, la dépêche, tec). L'autre pôle, on trouve l'implication, et l'article de commentaire dont la chronique, notre objet d'étude, fait partie.

\subsection{L'acte d'énonciation dans la chronique}

5 La chronique, à partir de laquelle nous avons relevé les créations lexicales à effet humoristiques, se présente comme étant

L'article dans lequel une « signature » rapporte ses observations, impressions et pas réflexions au fil du temps passé. [...] C'est en quelque sorte un journal d'auteur à l'intérieur d'un journal de journalistes. L'auteur en question, qui d'ailleurs peut être ou ne pas être un journaliste, a ses propres critères de sélection et d'appréciation du ou des sujets dont il désire s'entretenir selon son humeur. (J. De Broucker, 1995 : 207).

6 Selon le G-Blas ${ }^{8}$, la chronique est un des genres journalistiques qui « doit être courte et hachée, fantaisiste, sautant d'une chose à l'autre et d'une idée à la suivante sans la moindre transition. » Elle est un article écrit au jour le jour. Elle se présente comme un reflet de la vie quotidienne. Mais, qui selon M. Voirol, brasse plutôt les idées. La chronique en tant que genre journalistique a pour objet de dénoncer les travers de la société "de développer des idées, de livrer une opinion, d'affirmer une opposition.» (Voirol, 1995: 18) C'est un article de commentaire qui a pour objet de livrer une opinion, d'affirmer une position. C'est donc une lecture personnelle de l'actualité par son auteur et une façon de la raconter dans la plus grande liberté avec la bonne humeur et avec la légèreté de l'esprit. L'auteur précise que tout article de commentaire dont la chronique fait partie, est forcément « subjectif ».

7 Cette subjectivité se traduit par le fait que l'énonciateur ou le chroniqueur manifeste sa propre opinion ou ses propres appréciations dans l'analyse qu'il propose. Ce caractère subjectif se désigne aussi par l'ensemble des moyens linguistiques dont dispose le chroniqueur pour marquer sa présence dans l'énoncé. La présence de l'auteur se 
ressent non seulement dans les mots et les phrases qu'il utilise, mais même au niveau des phonèmes, et dans la ponctuation. Pour cela le chroniqueur utilise, selon D. Banks (2005: 260), la sublocution, néologisme, qui peut se diviser en : - modalisation, ou domaine de jugement (appréciatifs), (judicatifs) ou (contradictifs). - modulation, ou domaine de l'expressivité des divers sentiments du locuteur. Ces termes axiologiques sont utilisés pour pouvoir persuader le lectorat.

\subsection{Caractéristiques linguistiques de la chronique Tranche de vie}

8 Sur le plan linguistique, la chronique en tant que genre journalistique est également un genre littéraire car elle doit, selon J. Mouriquand (1997: 19), ses «caractéristiques à l'écriture, au talent de littéraire de l'auteur ». Production journalistique qui entretient des liens étroits avec la littérature, l'écriture de la chronique est souvent remarquable par la maîtrise de la langue. Rédigée souvent par des sociologues, des ethnologues, des anthropologues, la chronique est l'espace journalistique où écrivains peuvent s'introduire. Ceci dit que le chroniqueur n'est pas forcément un journaliste ou une personne qui a reçu une formation dans le domaine journalistique. C'est d'ailleurs le cas de l'auteur de la chronique Tranche de vie qui était avant tout un homme de théâtre9.

Pour ce qui est de la dimension linguistique et du style d'écriture adopté, le chroniqueur interpelle ses lecteurs dans une langue simple non spécialisée. Le chroniqueur traite des sujets dont il dévoile le contenu de chaque fait d'actualité, en s'intéressant à la fois à des thèmes variés pour en faire une critique dans une langue le plus souvent dérisoire et satirique, acquise avant toute chose à l'humour. L'auteur emploie également plusieurs tournures populaires et plusieurs parlers porteurs de sens et transformateurs du message voulu. Ainsi, le registre familier et le code oral ne sont pas épargnés dans ses chroniques où l'on assiste non seulement à l'utilisation de l'alternance codique comme dans le petit paragraphe suivant :

«...Pendant le Ramadhan, il y a beaucoup de baraka ${ }^{10}$. $\mathrm{Fi}^{11}$ l'entreprise même le planton devient charika gadra ${ }^{12}$. Et comment... c'est lui qui est devant la pointeuse... si tu veux pointer à ta guise, il te faut pointer chez le monsieur de la pointeuse... et koulchi yemchi ${ }^{13}$.» (22/08/2009).

10 Mais nous assistons également à la naissance d'un autre phénomène linguistique, phénomène de la création d'unités lexicales nouvelles hybrides formées de deux composants, l'un relevant de la langue française, l'autre des langues en présence dans l'univers algérien comme le montre bien le petit passage suivant :

...Au fait, les samedhan, dimandhan, lundhan, mardhan, mercredhan ${ }^{14}$, les jours du Ramadhan, on devient moderne, la technologie est utilisée à fond. On n'a pas besoin de se déplacer pour donner un ordre ou lancer une opération. Faire un suivi ou suivre une directive. Il suffit d'une connexion $\mathrm{ADSL}^{15}$ payée par la charika ${ }^{16}$, une puce, un bon téléphone mobile, et du champ et du champ. Sur le champ tout se règle...

\subsection{L'humour à travers les créations lexicales dans Tranche de vie}

11 Considérée comme le principal vecteur du changement linguistique, la presse écrite algérienne, en l'occurrence la chronique journalistique, est perçue comme une institution de la liberté d'information et d'expression dans le sens où elle constitue un lieu privilégié d'apparition et de création de mots nouveaux ${ }^{17}$. Les exemples cités plus haut illustrent parfaitement ce nouveau phénomène linguistique. Ceci symbolise sans 
doute cette capacité de la presse écrite à inventer la langue en créant des mots et des expressions. Manipuler la langue est donc une tâche aisée pour les chroniqueurs du moment qu'ils jouissent de cette qualité qui leur est attribuée en agissant surtout sur le lexique.

La création lexicale se fait naturellement pour dénommer des concepts et des nouvelles réalités (J. Pruvot, J-F Sablayrolles, 2003: 17). Ayant une fonction pratique, ces créations sont aussi utilisées pour augmenter l'expressivité du discours, pour inciter à la lecture et établir une certaine connivence et entretenir des rapports de familiarité avec le lecteur. Dans le cas des contextes médiatiques, le néologisme finit, par fois, par être utilisé comme une unité lexicale ordinaire. Une certaine complicité naît alors entre le quotidien et ses lecteurs. (L Sader Feghali, 2005 : p. 532). Cette connivence repose principalement sur la langue qui pourrait favoriser l'émergence des créations lexicales à effet humoristique. A l'égard de F. Evrard (1996:144) :

« l'écriture humoristique tend à manipuler le langage comme le lieu d'une activité ludique et poétique au sens de création, recréation et récréation ». Il s'agit donc de « créations délibérées » (J-F Sablayrolles, $2017:$ 37-50).

Créations instantanées et individuelles qui servent également à faire de l'humour. Construction de l'humour par des procédés de création lexicale, c'est donc l'une des stratégies discursives adoptée par les chroniqueurs qui consiste selon Charaudeau à :

S'affronter au langage, se libérer de ses contraintes, qu'il s'agisse des règles linguistiques (morphologie ou syntaxe) ou des normes d'usage (emplois réglés par des conventions sociales en situation), ce qui donne lieu au jeu de mots [...]. Et enfin elles demandent à un certain interlocuteur (individu ou auditoire) de partager ce jeu sur le langage et le monde, d'entrer dans cette connivence de "jouer en semble ", mais un jouer qui engage l'individu à devenir un autre, l'instant de l'acte humoristique, ce qui permet de dire que l'acte humoristique n'est jamais gratuit. $\mathrm{Au}$ total, l'humour correspond toujours à une visée ludique, mais à celle-ci peuvent s'adjoindre d'autres visées plus critiques, voire agressive... (P. Charaudeau, 2011 : 9-43)

Cet humour, nous le verrons, sous toutes ses formes (ironie, dérision et satire) serait donc un des moyens privilégiés du chroniqueur pour capter l'attention de ses lecteurs. C'est une façon humoristique de raconter le quotidien et refléter une image caricaturée des divers domaines de la réalité sociale. Celle-ci constitue pour la créativité langagière ${ }^{18}$ la source première de sa dynamique.

\section{Le corpus d'étude}

Notre corpus est constitué d'un ensemble d'exemples de créations lexicales recueillies par la méthode de dépouillement systématique de plusieurs chroniques journalistiques ${ }^{19}$. Pour pouvoir comprendre le phénomène de la créativité lexicale comme vecteur de l'humour que l'on entreprend de présenter d'abord et d'analyser par la suite dans la presse journalistique algérienne d'expression française, nous avons choisi comme corpus un échantillon des chroniques tirées de la presse non spécialiste mais généraliste telle que la chronique Tranche de vie du journal Le Quotidien d'Oran. Cette chronique bien connue, jouit d'une grande notoriété et est marquée par sa façon typique d'aborder des sujets variés qui reflètent la vérité et la réalité de notre société. Cette dimension a motivé une part, subjective, du choix de notre corpus. Nous avons aussi limité notre étude à cette rubrique dans la mesure où la chronique Tranche de vie, 
compte à elle seule un nombre important de créativités lexicales dont le versant linguistique est pris en ligne de compte.

Nous avons ainsi recensé un nombre assez considérable des créations lexicales. Notre source des créations lexicales s'est limitée aux années 2007-2016. Nous tenons à préciser que le choix de la période de la collecte des néologismes n'est pas pris au hasard, mais il s'agit bien d'un choix qui peut être qualifié de stratégique dans la mesure où la période détermine les conditions de création des mots. Nous relevons donc une relation entre l'emploi des néologismes et le contexte de la période.

15 J-F Sablayrolles précise que «le processus néologique n'est pas activé avec une intensité constante et régulière : certaines époques voient l'apparition de nombreux néologismes d'autre n'en voient apparaître que très peu. » (J-F Sablayrolles, 2000 : 133) En effet, la période détermine les conditions de création des mots. Nous relevons donc une relation entre l'emploi des néologismes et le contexte de la période. Ainsi Z. Xu (2001 : p. 55) affirme que

« l'analyse quantitative d'un corpus peut souvent servir à dégager une certaine tendance linguistique reliée au contexte d'une époque particulière dans une société donnée. ».

Les conditions particulièrement extralinguistiques qui ont favorisé l'émergence des créations lexicales à effet humoristique peuvent être liées aux évènements nationaux (sociaux, économiques, culturels) et internationaux (politiques, économiques, médicaux,...). Tous ces évènements qui ont marqué cette époque et ont fait d'elle une période propice à l'activité de création de nouveaux mots dans le domaine journalistique.

\section{Quels axes d'analyse?}

16 J-F Sablayrolles (2000 : 72) établit une typologie des typologies en s'inspirant largement des travaux de Tournier (1985 et 1991) qui pour sa part hiérarchise les néologismes selon deux matrices, une matrice externe et une matrice interne. J-F Sablayrolles (2000: 245) propose d'enrichir la classification de Tournier avec une typologie axée sur une sous-typologisation des matrices lexicogéniques. Cette typologie distingue la matrice externe (emprunt) des matrices internes en sous-catégorisant cette dernière selon quatre matrices : morphosémantiques, syntactico-sémantiques, morphologiques et phraséologiques. Pour notre étude, nous entreprenons l'analyse du corpus par l'application du dernier état du tableau des matrices lexicogéniques de l'année 2011 présenté en annexe à la page 09.

17 Nous partons donc de cette grille d'analyse qui gouverne le processus de formation des mots nouveaux. Par le biais de cette grille, nous entreprenons l'analyse de nouvelles unités lexicales produisant un effet humoristique. Subséquemment, il est important de rappeler que l'objectif assigné à cette étude est de mettre en lumière les procédés de création lexicale les plus privilégiés ( les procédés de dérivation, de composition, d'inversion, la synapsie, la troncation ou la siglaison, la métaphore, l'emprunt,...) pour véhiculer l'humour, et ce, en établissant le classement des innovations lexicales que nous avons relevées d'après leurs domaines d'utilisation ou le champ notionnel où s'applique avec humour la créativité lexicale. Ceci nous conduit aussi à nous rendre compte du domaine le plus accru aux créations lexicales comme vecteurs d'humour par rapport aux autres domaines. 


\section{Analyse lexicale et discursive des énoncés humoristiques}

18 L'examen du corpus ${ }^{20}$ a révélé que l'emploi des matrices internes ${ }^{21}$ semble intéressant en matière de création lexicale humoristiques dans les différents domaines. L'activité sociale avec ses divers domaines constitue pour la créativité langagière la source première de sa dynamique. A cet effet, il nous a semblé intéressant d'établir le classement des innovations lexicales que nous avons relevées dans notre corpus d'après leurs domaines d'utilisation ou le champ notionnel où la lexie néologique à effet humoristique apparaît.

19 Ainsi, la répartition des créations lexicales par domaines nous a permis de rendre compte de la domination du domaine faits et comportements sociaux. Plus que tous les autres domaines, le domaine social se prête à l'ironie. Notre corpus atteste que le discours attaché à la société est souvent ironique et l'ironie n'y est que partiellement cachée. Les constructions ironiques sont assez fréquentes. Etat de buvresse ${ }^{22}$, cette lexie inventoriée par le chroniqueur, illustre l'état d'une personne qui boit jusqu'à l'ivresse. Peut-on la considérer aussi comme une lexie appartenant aux matrices phraséologiques $^{23}$ ou lexie créée par détournement Sablayrolles J-F, (2009: 17-28.) de l'état d'ivresse? Le composé relié par l'interfixe-o-, mobiliotite $e^{24}$, otiteur ou encore otitophone ${ }^{25}$ créés par le chroniqueur, ne sont pas aussi sans une certaine charge ironique. C'est une manière de se moquer des personnes qui ont tout le temps leurs oreilles collées contre leurs téléphones portables. Ils ont été créés pour désigner ironiquement un phénomène social très répandu chez les jeunes. La juxtaposition de deux mots constitue un oxymore tel que mendicité new-look. Ce composé hybride a été inventé pour désigner les jeunes joliment habillés et qui demandent l'aumône. Un autre néologisme à effet humoristique, pneumanie, a été formé par analogie à pneumonie indiquant les citoyens qui brûlent des pneus pour exprimer leur colère et leur mécontentement.

20 Le domaine de la culture, avec toutes ses variétés, étant donné sa nature très large et l'apport personnel du chroniqueur, se trouve plus au moins accru par rapport aux autres domaines. L'émergence de ce phénomène dans les chroniques journalistiques peut se rattacher également à un grand évènement culturel qui s'est produit à Alger. Le Panaf ${ }^{26}$, l'une des plus grandes manifestations culturelles d'Afrique où intellectuels et artistes africains se sont réunis en juillet 2009. En effet, la synapsie panne à fric a été créée pour dénoncer les dépenses onéreuses consenties à ce festival. Des néologismes à effet humoristique liés à ce domaine ont aussi été trouvés tels que téléaste $e^{27}$, skechistes ${ }^{28}$, écriveur ${ }^{29} \mathrm{ou}$ encore cultureurs ${ }^{30}$ pour ridiculiser ceux qui manquent de culture civique.

21 Il est également important de dire que la langue n'existe pas séparément de la culture. En effet, la langue n'est qu'un aspect d'une culture. Notre corpus confirme ce constat. La majorité des emprunts est faite à l'arabe, qui est omniprésent dans le domaine culturel. Ce dernier a enregistré un nombre considérable de lexies néologiques dont la majorité est empruntée à la langue arabe notamment l'arabe dialectal. De ce fait, nous nous rendons aisément compte de la domination de l'arabe comme première source d'emprunts. Le chroniqueur les utilise pour décrire un évènement culturel spécifique à la réalité algérienne. Ainsi, la chronique journalistique semble se caractériser par un certain effort d'employer des emprunts hybrides. Notre corpus atteste ce procédé de 
création par hybridation faisant recours surtout à l'emprunt. Des hybrides comme hardrai $^{31}{ }^{31}$ jazz karkabou ${ }^{32}$ ont été recensées dans notre corpus. La création lexicale, comme phénomène linguistique au service du rire et d'amusement, paraît réussie dans ce cas mais la nouveauté n'est pas portée par les mots dans leur isolement, mais bien dans leur association.

Le domaine politique est susceptible d'avoir recours aux créations lexicales à effet humoristique. La lexie foot politique a été créée pour refléter les relations entre l'Algérie et l'Egypte qui, depuis le match qui s'est déroulé dans le cadre des éliminations de la coupe du monde 2010, sont devenues tendues. Au sujet de la politique extérieure, la lexie doigtalisme ${ }^{33}$ a été créée par le chroniqueur. Pour lui, c'est un concept qui est né avec le printemps arabe 2010. "C'est grâce à cette méthode de vote (le doigtalisme) que des pays, démocratiquement, ont élu ceux qui les mènent en bateau, aujourd'hui.» (5/07/2014). La politique intérieure est très évoquée. Ainsi l'amalgame dictarchie combine les fragments des mots «dictature » et "anarchie», le chroniqueur l'a crée pour désigner, d'une manière satirique, deux systèmes sociopolitiques en principe antagonistes qu'il associe pour décrire sa vision de l'Algérie. Parlons du même domaine, des composés reliés par l'interfixe-o ont été créées pour décrire par manière de dérision le système politique en Algérie. A titre d'exemple, nous citons ces quelques compositions plus moins exagérées : Politicopolitiques ${ }^{34}$, historicopoliticotactique, tecnicotacticofootbalisticopolitique.

Attachées au domaine économique, des lexies à effet humoristique ont été créées telles que dinariste $e^{35}$, se milliardérisé, se dolarisant ou encore grippe financière ${ }^{36}$. Cette dernière création a été obtenue par altération phonétique, ne renvoie pas à grippe mais à la crise financière qui a touché le système économique de tous les pays en particulier l'Algérie. Par comparaison avec les autres domaines, la technologie n'intervient toutefois que très peu dans la néologie journalistique. Car la terminologie, selon J-C Corbeil (1974), ne semble pénétrer que lentement dans la presse générale. Il est à remarquer que dans le cas des innovations lexicales à caractère scientifique ou technique, la presse recourt surtout à la vulgarisation. Nous pouvons citer l'exemple suivant : « La NASA, encore elle, a déjà commencé à étudier le concept d'un recyclage. L'idée consistait à développer une PaCM (pile à combustible microbienne ultra-compacte)...». Néanmoins, des innovations ont été inventées pour dénommer par manière de dérision. Parlons de nouvelles technologies, le chroniqueur s'amuse à créer des lexies telles que otitophone ou encore mobiliotite. Selon le chroniqueur, il s'agit d'une otite qu'on peut avoir à cause du téléphone portable.

En ce qui concerne le domaine religieux, les créations lexicales à effet humoristique n'ont pas été recensées. Ce domaine n'est pas propice à la créativité. Cette abstinence à la création est due, pensons-nous, à la nature même du domaine. En d'autres termes, le domaine religieux, où ne s'applique pas avec humour la créativité lexicale, est un domaine traditionnellement sacré et donc n'est pas favorable à la création. Le chroniqueur préfère s'abstenir de faire des créations dans ce domaine. L'analyse discursive de la presse écrite francophone, en l'occurrence la chronique Tranche de vie du journal Le quotidien d'Oran, a essentiellement porté sur le processus de la création des lexies comme vecteur de l'humour. Ces lexies, volontairement créées, sont insérées dans un contexte à caractère humoristique afin d'établir avec le lecteur une certaine familiarité et connivence. Ces dernières se manifestent par l'usage de la langue sous toutes ses formes : alternance des langues, emprunt à l'arabe dialectal, en particulier, jeux de mots et création lexicale qui, contribuent en grande partie à l'effet 
humoristique. Parmi les procédés de création lexicale, certains, comme la dérivation, la composition, la synapsie, la métaphore..., sont privilégiés pour véhiculer l'humour.

Ainsi, en lisant ces créations, on voit bien que le genre de la chronique est plutôt ironique. L'humour dans toutes ses formes (ironie, dérision et satire) est donc une des stratégies que le chroniqueur adopte pour révéler le quotidien. Le chroniqueur se présente ainsi comme un facilitateur de lecture des réalités sociales. C'est une façon donc humoristique et satirique de dévoiler le quotidien et refléter une image caricaturée de la réalité sociale. L'activité sociale avec ses divers domaines constitue pour la créativité langagière la source première de sa dynamique.

La répartition des créations lexicales à effet humoristique par domaines nous a permis de rendre compte de la domination du domaine social. Le domaine culturel se trouve plus au moins néologène par rapport aux autres domaines. Fait confirmé par le nombre considérable des emprunts. Ainsi des lexies telles que cultureur ou encore sketchistes ont été recensées. Il est à noter ensuite que le domaine politique vient en troisième position. Or, des créations lexicales attachées à la politique intérieure sont plus nombreuses que celles qui appartiennent à la politique extérieure. Des lexies néologiques ont été également recensées dans le domaine économique. En effet, ces innovations lexicales à effet humoristique ont été créées suite à la crise économique qui a touché le système économique de tous les pays du monde. Le domaine scientifique et de la technologie vient en dernière position. Cela s'explique par le fait que nous avons affaire à un journal non spécialisé.

Cette étude qui s'est intéressée à l'analyse de l'acte humoristique par le biais des procédés de création lexicale dans les chroniques journalistiques, ne prétend pas l'exhaustivité. Bien certainement, tous les résultats ainsi que les remarques ne sont valables que pour notre corpus d'étude. Bien qu'ayant recherché, dans cette étude, à examiner le mieux possible notre corpus, il peut néanmoins contribuer à approfondir d'autres pistes de recherche: une analyse approfondie qui permet de voir comment s'applique avec humour la création lexicale dans le discours journalistique en constituant des corpus plus larges et plus diversifiés.

\section{BIBLIOGRAPHIE}

ADAM J-M., (1997), « Unités rédactionnelles et genres discursifs : cadre général pour une approche de la presse écrite ", In Pratiques, $n^{\circ}$ 94, p. 3-18.

ALLAM-IDDOU S. \& BOUTMGHARINE N., (2016), « Le français d'Algérie et du Maroc : une étude comparative des interférences comme source de créativité lexicale ». Université de Paris Diderot. . Colloque International Langues, Cultures et Médias en Méditerranée : formes, sens et développemen. <hal-01370321>.

BANKS D., (2005), Les Marqueurs Linguistiques De La Présence De L'auteur, L'Harmattan. p. 260. 
CATARING A-T., (2011), « Néologismes d'auteurs »dans la presse écrite généraliste. http:// www.contrastiva.it /.../ Deroy, \% 20Tipologie \%20general \%20neologis. Site consulté le $20 / 11 / 2011$.

CHARAUDEAU P., (2011), « des catégories pour l'humour. Précisions, rectifications, compléments. ", In Vivero Ma.D. (dir.), Humour et crises sociales. Regards croisés France-Espagne, (pp. 9-43), L'Harmattan, Paris, Article disponible sur URL : http:// www. Patrick Charaudeau. com/Des-categories-pour-l-humour, 274.html, Consulté le 02/05/2013.

CHARAUDEAU P., (1997), Le discours d'information médiatique, la construction du miroir social, coll. Médias-Recherches, Nathan, Paris.

CORBEIL J-C., (1974), « Analyse des fonctions constructives d'un réseau de néologie, l'aménagement de la néologie », actes du colloque international de terminologie, office de la langue française, Québec, mai 1975.

CORBIN D., (ed. 1991), La formation des mots : structures et interprétations, (Lexique, 10.) Lille : Presses universitaires de Lille, 294 pp. 290717004 X.

DE BROUCKER J., (1995), Pratique de l'information et écritures journalistiques, p. 207,CFPJ, Paris.

EVRARD F., (1996), L'humour, « Contours Littéraires », Hachette

MOURIQUAND J., (1997), L'écriture journalistique, p. 19, PUF, Paris.

PICARD J.-C., (1999), « La chronique dans les quotidiens québécois : un genre journalistique de plus en plus populaire », in Les Cahiers du journalisme, $n^{\circ} 6$, 1999, pp. 36-49. Disponible sur le site : http://www.cahiersdujournalisme.net/cdj/pdf/06/06_Picard.pdf.

PRUVOST J., SABLAYROLLES J-F., (2003), Les néologismes, n 3674, p. 17, Paris, « Que sais-je ?», PUF, 128 pages.

SABLAYROLLES J-F., (2000), La néologie en français contemporain. Examen du concept et analyse de productions néologiques récentes, p.p72-133, Honoré Champion

SABLAYROLLES J-F., (2009), « Des néologismes par détournement? ou Plaidoyer pour la reconnaissance du détournement parmi les matrices lexicogéniques ", Recherches, didactiques, politiques linguistiques : perspectives pour l'enseignement du français en Italie, Oct 2009, Milan, France. pp. 17-28. ffhalshs00735933f https://halshs.archives-ouvertes.fr/halshs-00735933 Consulté le [12/5/2013].

SABLAYROLLES J.-F., (2017), « Créativité lexicale en discours liée à l'existence de paradigmes », in Signata, Annales des sémiotiques/ Annals of semiotics, Article disponible en ligne à l'adresse : https://journals.openedition.org/signata/1345

SADER FEGHALI L., (2005), « La presse vue à travers Néoscope : Quand les contextes médiatiques sont mis au service de la néologie ", In Mots, Termes et Contextes, Actes des septièmes Journées scientifiques du réseau de chercheurs, Lexicologie Terminologie Traduction, Bruxelles, Belgique-8, 9 et 10 septembre, p. 532.

VOIROL M., (1995), Guide de la rédaction, p. 18, CFPJ, Paris.

Xu Z., (2001), Le néologisme et ses implications sociales, p. 55, L'Harmattan. 


\section{NOTES}

1. Pour comprendre comment le phénomène de l'innovation lexicale humoristique que l'on entreprend de présenter d'abord et d'analyser par la suite, nous avons choisi comme corpus un échantillon des chroniques publiées dans le journal quotidien généraliste algérien en langue française Le Quotidien et le journal.

2. Issu d'un nom d'un homme politique, le président Bouteflika, création par analogie (à marxisme), cette lexie indique une attitude politique. Construction hybride, cet exemple illustre aussi la tendance à l'association de la lexie arabe (nom d'un patronyme) à un morphème de la langue française.

3. Cette création combine les fragments de mots " démocratie » et " anarchie » désignant deux systèmes sociopolitiques en principe antagonistes. Il s'agit sans doute de la démocratie pratiquée d'une façon anarchique.

4. Cette lexie est construite sur un nom propre Bill Gates. Elle a été créée à l'occasion de l'Aïd, fête religieuse musulmane, fête du sacrifice pour désigner les vendeurs de troupeaux qui font fortune en cette occasion et il les compare alors à Bill Gates.

5. Il s'agit d'une une conversion $\mathrm{N}->\mathrm{V}$ sur un nom emprunté. Il a été créé pour désigner le comportement des femmes qui changent de tenue dans un mariage, le chroniqueur les compare aux artistes.

6. Le processus sémantique néologique est créé essentiellement à partir de métaphores comme le Lénine du Funk. La star américaine du genre musical « funk », Mikael Jackson, est assimilée à une figure politique russe, Lénine, associée à la révolution russe d'octobre 1917.

7. Pour pouvoir analyser les lexies néologiques, il est nécessaire de ne pas s'arrêter seulement à la création lexicale mais de prendre en considération "l'ensemble de la situation de production et extra-linguistique »Chr Marcellesi (1974:95-96), des néologismes, qui sont à saisir et à étudier dans leur contexte (le texte dans lequel ils apparaissent) et l'environnement général. On relève donc une relation entre l'emploi des néologismes et le contexte de la période.

8. Un quotidien de la presse écrite française (1879-1938/1940) dans lequel Maupassant écrit Messieurs de la chronique, novembre 1889.

9. Dans la chronique journalistique Tranche de vie apparait le pseudonyme de l'auteur soussigné EL Guellil, dont le vrai nom est Baba Hamed Fodil. A treize ans, il était déjà au théâtre amateur. Plus tard, il se transporte à Alger pour poursuivre des études de théâtre à l'Institut nationale des Arts dramatique. Ensuite, il passe un bref séjour de quelques années à l'école des arts et métiers de Paris. Puis, il retourne travailler en Algérie dans l'entreprise Nationale de Cinéma. Dans les années 1990, la libération du champ médiatique, avec la création de la presse privée, il a été un des pionniers de la presse privée à Oran avec la création d'un journal du soir puis l'Espoir et enfin le journal Le Quotidien d'Oran où il occupe le poste de directeur technique en plus de l'animation quotidienne de la rubrique Tranche de vie. En 2006, ses chroniques ont été rassemblées dans un recueil de 293 pages, sous le titre Tranche de vie par el-Guellil, publié par la maison d'Edition Dar el Gharb d'Oran.

10. La bénédiction est la traduction en français.

11. Dans est la traduction en français.

12. Cela veut dire une grande entreprise en français mais dans ce passage, cette expression veut dire un homme riche.

13. Tout est en marche.

14. Cette lexie et les autres sont complexes car elles sont fabriquées à partir de la lexie dimanche, lundi, mardi, mercredi et la lexie arabe Ramdhan, le mois du jeûne pour les musulmans. Ces néologisme ont été crées sur le modèle de formation apocope (diman, lund, mard, mercred) et aphérèse (dhan) en utilisant le procédé de compocation. Ils désignent les jours du Ramadhan.

15. Protocole de transmission numérique à haut débit qui utilise le réseau téléphonique. 
16. Entreprise.

17. Ce phénomène de la création lexicale de la presse témoigne de l'évolution de la société algérienne depuis le début des années 1980. Après la décennie noire, les libertés d'opinion et d'expression ont fait de la période transitoire (1988-1990) une période propice à l'émergence de nouveaux mots dans les médias indépendants. Cette mutation médiatique a eu une incidence directe sur la dynamique et l'évolution des pratiques linguistiques des journalistes. Certaines chroniques journalistiques des années 2007-2016 ont suscité l'intérêt que nous portons à la création lexicale.

18. Nous avons également pris en considération le cotexte qui indique le groupe de termes dans lequel se trouve la création lexicale, pour aussi déterminer la catégorie grammaticale du néologisme.

19. Corpus recueilli dans le cadre d'une recherche menée en 2009-2015, qui s'inscrit dans la thèse de doctorat qui s'intéresse à la néologie journalistique et en particulier l'innovation lexicale et la productivité des procédés de nouvelles créations lexicales dans la presse francophone algérienne, soutenue et mise ligne, en 2017, sur le site: http://e-biblio.univ-mosta.dz dirigée par J-F Sabalyrolles.

20. La liste des créations, présente ici, n'est pas exhaustive.

21. Nous développerons ultérieurement l'analyse détaillée des créations appartenant à ces matrices morphosémantiques, syntaxico-sémantiques, morphologiques pragmatico-sémantique ainsi que la matrice externe. Celle-ci est principalement constituée de procédés relevant de l'interférence tels que l'emprunt.

22. Cette lexie est issue du participe passé bu du verbe boire et ivresse, état d'une personne ivre.

23. Se référer au tableau intitulé annexe, p. 12.

24. Pour créer ce mot composé, le chroniqueur a pu relier par l'interfixe-o- deux mots mobile et otite.

25. Ce nom d'agent est construit sur la base otite et le suffixe -eur. Ce néologisme a été crée pour désigner ceux qui ont le téléphone portable collé à l'oreille: "Comme tous les "otiteurs" (otiteur, c'est celui qui, la journée durant, a son portable collé à l'oreille) [...] »

26. Festival Panafricain d'Alger.

27. Par analogie à cinéaste

28. Cette lexie a été créée par analogie à humoriste.

29. C'est un dérivé du verbe écrire qui laisse supposer une connotation sémantique péjorative de ce mots dérivé désignant l'écrivain pauvre: [non, il ne s'agit pas du trou du journal que l'infortuné écriveur, que je suis [...]

30. cultureur constitue pertinemment une lexie néologique par affixation. La comparaison de cultureur et cultivé ou culturel permet de relever la valeur péjorative de ce suffixe dans la formation de ces dérivés.

31. Lexie néologique par analogie à Hard Rock.

32. Des composés hybrides où les deux éléments constitutifs appartiennent à deux langues différentes, anglais/arabe.

33. Doigtalisme vs au vote informatisé.

34. politico-politique s'assimile plutôt à un pléonasme même si le composant politico ajoute une détermination supplémentaire à politique puisqu'il en spécifie, pensons-nous, le type de système politique établi en Algérie.

35. Cette lexie et la suivante ont été inventées d'une manière ironique pour décrire la situation économique d'une minorité d'Algériens ont pu faire de l'argent au détriment de certains d'autres qui ne peuvent même pas subvenir aux besoins les plus élémentaires de leur famille. : «Depuis qu'une minorité de superalgériens, en se « dinarisant » à outrance et en se "dollarisant » avec aisance, s'est « milliardisée » au détriment des « ah j'ai rien!» 
36. Il s'agit de la déformation volontaire du signifiant par jeu de mots. Cette lexie est créée ironiquement: «Moi, je vous le dis, quitte à vous paraître ridicule, que la crise porcine et la grippe financière qui affectent toute la planète ne peuvent pas faire de dégâts chez nous car on est immunisés [...]»

\section{RÉSUMÉS}

Notre contribution vise à mettre en valeur l'acte humoristique dans le discours journalistique francophone à travers des créations lexicales. Si l'innovation lexicale a fait l'objet de nombreuses études, nous ne recensons aucune étude consacrée à la création lexicale à effet humoristique de la presse algérienne francophone. Pour cela, nous nous permettons d'actualiser une analyse discursive de l'humour à travers les créations lexicales manifestées dans le discours journalistique. Dans le cadre de cette étude, nous proposons tout d'abord d'identifier toutes les créations pouvant servir de base à des effets humoristiques afin de les analyser puis nous mettons en évidence la mise en œuvre du processus de la création lexicale.

Our contribution aims to highlight the humorous act in French-speaking journalistic discourse through lexical creations. While lexical innovation has been the subject of much study, we haven't found any studies devoted to the humorous neology of the French-speaking Algerian press. To this end, we allow ourselves to update a discursive analysis of humor through the lexical creations manifest in journalistic discourse. In the framework of this study, we first propose to identify all the creations that can serve as a basis for humorous effects in order to analyze them, then we highlight the implementation of the lexical creation process.

\section{INDEX}

Mots-clés : Créativité lexicale, acte humoristique, processus de formation de mots, presse écrite algérienne, processus de création lexicale

Keywords : Lexical creativity, humorous act, word formation processes, Algerian written press, lexical creation processes

\section{AUTEUR}

\section{SAMIRA ALLAM IDDOU}

Laboratoire de rattachement ELILAF : Université de Mostaganem, Algérie 